Conclusion These data suggest a high burden of TV and co-infection with CT. Public health surveillance focusing on CT infection may consider routine screening of other STIs, including TV, in local populations to reduce STI morbidity.

\section{P3.348 MATERNAL AND CONGENITAL SYPHILIS REMAIN A CHALLENGE}

doi:10.1136/sextrans-2013-051184.0801

1.2D M S Magalhães, 'A Dias, II M P Calderon. 'Programa de Pós-graduação em Ginecologia, Obstetrícia e Mastologia da Faculdade de Medicina de Botuc, Botucatu, São Paulo/SP, Brazil, ' 2 Secretaria de Estado de Saúde do Distrito Federal, Brasília, Brazil

This descriptive study set the profile of pregnant with positive VDRL followed at public maternity hospitals in Federal District, Brazil, of the newborns children of mothers with syphilis that showed clinic signs of congenital disease and verified approach according with the recommendations of the Ministry of Health. The study verified the percentage of pregnant women who had access to prenatal care and were inadequately treated and the percentage of newborns with inadequate clinical management in accordance with the recommendations of the MS. We interviewed 67 pregnant as postpartum women who reported to the National System of Disease Notification, users of five public hospitals of the DF and consulted information from medical records and from prenatal care charts, when available. The information relating to the clinical and laboratory received to the children were supplemented with data from medical records, compulsory notification forms of syphilis, from prenatal care charts and the child health card, when available. Data were collected on sociodemographic, obstetric, information related to diagnosis, treatment of pregnant and puerperal women their partners and treatment of newborns into delivery. Only $41.8 \%$ patients were adequately treatment and main reason for inadequacy was the lack of treatment $(83.6 \%)$ or inadequate treatment of the partner $(88.1 \%)$. The study demonstrated the need for new treatment of the mother at the hospital for lack of documentation of the treatment in prenatal care. There was disagreement with the recommendations of the MS to follow-up of newborns with congenital syphilis in relation to radiographic study only $48 \%$ children carried out and lumbar pucture was required only $42 \%$. It was observed that $36 \%$ newborns not received any treatment. Our date demonstrates that the quality of prenatal care received by pregnant women is not sufficient to ensure control of congenital syphilis an reaching the goal of controlling the disease.

\section{P3.349 EPIDEMIOLOGICAL AND CLINICAL ASPECTS OF CONGENITAL SYPHILIS IN BELARUS}

doi:10.1136/sextrans-2013-051184.0802

0 Pankratov. Belarusian Medical Academy of Postgraduate Education, Minsk, Belarus

In the years 1996-2012 all together 4966 pregnant women infected with syphilis were registered in Belarus $(8.36 \%$ from the general number of the women with syphilis for this period - 59426 cases). Primary syphilis was diagnosed in $13.2 \%$ of patients, secondary syphilis - 30.5\%, latent early syphilis - $55.9 \%$, latent late syphilis $0.4 \%$. Disease has been revealed in I trimester of pregnancy in $56.7 \%$ of patients, II trimester $-23.2 \%$, III trimester $-17.5 \%$, after delivery $-2.6 \% .45 .1 \%$ of women have made abortion after disease revealing.

There are 127 children with congenital syphilis (CS) registered in 1994-2012 in Belarus in total, but last two years cases of CS have not been registered. Among women which have given birth to children with CS primary syphilis was not diagnosed, secondary syphilis $-26.7 \%$, latent early syphilis $-71.4 \%$, seroresistant syphilis $-1.9 \%$. Reinfection was registered in $8.5 \%$ of patients.
Early CS with symptoms (Table) has been revealed in $24(18.9 \%)$ of children, early latent CS - in $81.1 \%$. Cases of late CS have not been registered. Three children have died within the first month of a life.

Abstract P3.349 Table 1 Table-Clinical symptoms of manifest early CS in Belarus in 1994-2012 $(n=24)$

\begin{tabular}{lll}
\hline Symptom & Number of cases & $\%$ \\
\hline Pathology of bones: & 16 & 66.7 \\
osteochondritis II-III & 11 & 45.8 \\
periostitis & 1 & 4.2 \\
gummas of bones & 2 & 8.3 \\
pseudoparalysis of Parrot & & \\
Visceral pathology: & 17 & 70.8 \\
hepatosplenomegaly & 5 & 20.8 \\
hepatomegaly & 9 & 37.5 \\
congenital pneumonia & 2 & 8.3 \\
syphilitic nephritis & 2 & 8.3 \\
congenital heart disease & 1 & 4.2 \\
fibrosis of kidneys & 1 & 4.2 \\
fibrosis of adrenals & & \\
Pathology of nervous system: & 10 & 41.7 \\
encephalopathy & 3 & 12.5 \\
meningocephalitis & 2 & 8.3 \\
meningitis & 1 & 4.2 \\
hydrocephalus & & \\
Papules of skin and mucosas & 10 & 41.7 \\
Syphilitic rhinitis & 8 & 33.3 \\
Including with development of «saddle» nose & 1 & 4.2 \\
Syphilitic pemphigus & 5 & 20.8 \\
Roseola & 4 & 16.7 \\
Hochsinger's popular infiltration & 2 & 8.3 \\
Pathology of eyes: & 1 & 4.2 \\
syphilitic chorioretinitis & & 4.2 \\
syphilitic uveitis & & \\
\hline & & \\
& & \\
\hline
\end{tabular}

\section{P3.350 EVALUATION OF A COMPARATIVE WESTERN BLOT METHOD FOR EARLY POSTNATAL DIAGNOSIS OF CONGENITAL SYPHILIS}

doi:10.1136/sextrans-2013-051184.0803

'A Marangoni, 'P Nardini, , $\mathrm{C}$ Foschi, ${ }^{1} \mathrm{M}$ Compri, ${ }^{2} \mathrm{~A}$ Moroni, ${ }^{1} \mathrm{R}$ Cevenini. 'University of Bologna, Microbiology, DIMES, Bologna, Italy; ${ }^{2}$ S. Orsola Hospital, Microbiology, Bologna, Italy

Background Diagnosis of congenital syphilis (CS) remains difficult. Part of the problem arises because the standard serologic tests are not useful in newborns because IgG transfer across the placenta.

Since Western Blot technique allows the recognition of a specific response towards every single protein, it can be useful to compare IgG immunological profiles of mothers and babies at birth, in order to differentiate between passively transmitted maternal antibodies and antibodies synthesised by the infants.

\section{Methods}

Study group. Thirty infants born to syphilis seropositive mothers were enrolled for this study. At birth, routine serological tests were performed (ARCHITECT ${ }^{\circledR}$ Syphilis TP, Abbott; TPHA and RPR, Randox) on mother/child pairs' serum specimens.

"Home made WB". Treponema pallidum antigens, separated by SDS-PAGE, were blotted onto nitrocellulose sheets and incubated overnight with mother/child pairs' serum specimens.

Criteria for CS diagnosis were the following: presence of specific bands in the newborn's IgG WB strip different from those found on the corresponding maternal WB strip and/or recognition on IgM WB strip of at least 2 out the 4 following bands Tp47, TmpA, Tp17 and Tp15, including at least one with low molecular weight. 